\title{
How does electron beam irradiation dose affect the chemical and antioxidant profiles of wild dried Amanita mushrooms?
}

Running title: Chemical and antioxidant profiles of electron beam irradiated Amanita

Ângela Fernandes ${ }^{\mathrm{a}, \mathrm{b}}$, João C.M. Barreira, ${ }^{\mathrm{a}, \mathrm{b}}$, Amilcar L. Antonio ${ }^{\mathrm{a}}$, Andrzej Rafalski ${ }^{\mathrm{c}}, \mathrm{M}$. Beatriz P.P. Oliveira ${ }^{\mathrm{b}}$, Anabela Martins ${ }^{\mathrm{a}}$, Isabel C.F.R. Ferreira ${ }^{\mathrm{a},{ }^{*}}$

${ }^{a}$ Centro de Investigação de Montanha (CIMO), ESA, Instituto Politécnico de Bragança Campus de Santa Apolónia, Apartado 1172, 5301-855 Bragança, Portugal.

${ }^{\mathrm{b}}$ REQUIMTE/Depto. de Ciências Químicas, Faculdade de Farmácia, Universidade do Porto, Rua Jorge Viterbo Ferreira n. ${ }^{\circ}$ 228, 4050-313 Porto, Portugal.

${ }^{\mathrm{c} C e n t r e}$ for Radiation Research and Technology, Institute of Nuclear Chemistry and Technology, Dorodna str. 16, 03-195Warsaw, Poland

*Author to whom correspondence should be addressed (I.C.F.R. Ferreira: iferreira@ipb.pt, tel. $+351-273303219$, fax $+351-273325405)$. 


\begin{abstract}
As all the mushrooms, Amanita species experiment several conservation problems, with a post-harvest life limited to a few days. Drying is one of the most used methods in mushrooms preservation. Food irradiation is another possible way to improve food quality and insure its security. Among the emerging irradiation technologies, electron beam has wide application, allowing high throughput, wide flexibility and potential, without any negative effect on the environment. The effects of different electron beam irradiation doses in Amanita genus, were assessed by measuring the changes produced on a wide variety of nutritional, chemical and antioxidant indicators. The evaluated profiles indicated differences among non-irradiated and irradiated samples, but a high similarity among different doses. This finding advises the highest assayed dose (10 kGy), ensuring higher effectiveness from the decontamination and disinfestation point of view, without having stronger effects than those observed for the lower doses.
\end{abstract}

Keywords: Amanita spp.; Electron beam; Chemical composition; Antioxidant properties; Principal Component Analysis. 


\section{Introduction}

The post-harvest life of mushrooms is limited to a few days, due to their fast quality devaluation (Lukasse \& Polderdijk, 2003). After harvesting, moisture loss, shrinkage and rapid spoilage in terms of color and texture occur. Normally, mushrooms are consumed in fresh, but in recent years their consumption in dried forms has been increasing (Şevik, Aktaş, Doğan, \& Koçak, 2013). Drying is one of the oldest methods for preservation of food commodities for long duration and also one of the most used conservation methods employed in storage of mushrooms (Ma, Chen, Zhu, \& Wang, 2013).

The drying process causes the reduction of vegetative cells of microorganisms, which gives rise to a flora of bacteria and fungi that have the ability to survive for long periods in dried foods and produce toxins harmful to human health (ICMSF, 1985); the high humidity that exists during storage also predisposes dried mushrooms to invasion by microorganisms (Shephard, 2008; Ezekiel et al., 2013).

The prevention of food deterioration and the control of infection by microorganisms have been a major preoccupation of man over the centuries (ICGFI, 1999). Accordingly, food irradiation is one of the possible ways to improve the quality, reduce the incidence of foodborne diseases caused by microorganisms, decontaminate pests, insects or parasites that inflict food spoilage and toxicity, thereby replacing the chemical treatments (Supriya, Sridhar, Nareshkumar, \& Ganesh, 2012; Culleré, Ferreira, Venturini, Marco, \& Blanco, 2012). Among the emerging technologies of irradiation, electron beam (EB) has wide applications in improvement of food quality and safety; this technology is highly attractive due to its technological advantages in terms of high throughput, wide flexibility and potential, use of a non-thermal process, leaving no toxic residues and without any negative effect on the environment (Supriya, Sridhar, Nareshkumar, \& Ganesh, 2012). 
Our research group has been demonstrating that EB irradiation does not significantly alter the antioxidant, chemical and nutritional parameters of different mushrooms species namely, Macrolepiota procera (0.5, 1 and 6 kGy) (Fernandes et al., 2013a), Boletus edulis and Russula delica (2, 6 and $10 \mathrm{kGy}$ ) (Fernandes et al., 2014). The aim of the present study was to validate the use of $\mathrm{EB}$ irradiation preservation to other mushrooms species, maintaining their nutritional and chemical quality, as also antioxidant potential. Amanita caesarea (Scop.) Pers. and Amanita curtipes E.-J. Gilbert were irradiated at 2, 6 and 10 $\mathrm{kGy}$, doses typical used for mushrooms decontamination and conservation.

\section{Materials and methods}

\subsection{Standards and reagents}

For chemical analyses: Acetonitrile 99.9\%, n-hexane 95\% and ethyl acetate $99.8 \%$ were of HPLC grade from Lab-Scan (Lisbon, Portugal). The fatty acids methyl ester (FAME) reference standard mixture 37 (standard 47885-U) was purchased from Sigma (St. Louis, MO, USA), as also other individual fatty acid isomers, organic acids, tocopherols and sugar standards.

For antioxidant potential analysis: 2,2-diphenyl-1-picrylhydrazyl (DPPH) was obtained from Alfa Aesar (Ward Hill, MA, USA). Standards trolox (6-hydroxy-2,5,7,8tetramethylchroman-2-carboxylic acid) and gallic acid were from Sigma (St. Louis, MO, USA). Methanol and all other chemicals were of analytical grade and obtained from common sources. Water was treated in a Milli-Q water purification system (TGI Pure Water Systems, Greenville, South Carolina, USA).

\subsection{Samples and electron beam irradiation}


A. caesarea and A. curtipes samples were obtained in Trás-os-Montes, in the Northeast of Portugal, in October 2013, and dried at $30{ }^{\circ} \mathrm{C}$ in an oven. Subsequently, the samples were divided in four groups with five specimens of each mushroom species: control (non-irradiated, $0 \mathrm{kGy}$ ); sample 1 (2 kGy); sample 2 (6 kGy) and sample 3 (10 kGy), kept in polyethylene bags.

The irradiation was performed at the INCT - Institute of Nuclear Chemistry and Technology, in Warsaw, Poland. To estimate the dose during the irradiation process three types of dosimeters were used a standard dosimeter, graphite calorimeter, and two routine dosimeters, Gammachrome YR and Amber Perspex, from Harwell Company (UK). The irradiation took place in e-beam irradiator of $10 \mathrm{MeV}$ of energy with pulse duration of 5.5 $\mu$ s, a pulse frequency of $440 \mathrm{~Hz}$, and an average beam current of $1.1 \mathrm{~mA}$; the scan width was $68 \mathrm{~cm}$, the conveyer speed was settled to the range $20-100 \mathrm{~cm} / \mathrm{min}$ and the scan frequency was $5 \mathrm{~Hz}$. The estimated absorbed doses were 2.5, 6.2 and $10.9 \mathrm{kGy}$, with an uncertainty of 20\%. To read Amber and Gammachrome YR dosimeters, spectrophotometric methods were used at $603 \mathrm{~nm}$ and at $530 \mathrm{~nm}$, respectively, to estimate the dose from the value of absorbance according to a previous calibration curve. For the Graphite calorimeter dosimeter the electrical resistance was read and converted in dose according to a previous calibration curve (Fernandes et al., 2013a). For simplicity, we refer to the irradiation doses as: $0,2,6$ and $10 \mathrm{kGy}$.

Before analysis, the samples were reduced to a fine dried powder (20 mesh) and mixed to obtain homogenized samples.

\subsection{Chemical parameters}

\subsubsection{Nutritional value}


Moisture, protein, fat, carbohydrates and ash were determined following the AOAC procedures $($ AOAC, 1995). The crude protein content $(N \times 4.38)$ of the samples was estimated by the macro-Kjeldahl method; the crude fat was determined by extracting a known weight of powdered sample with petroleum ether, using a Soxhlet apparatus; the ash content was determined by incineration at $600 \pm 15{ }^{\circ} \mathrm{C}$. Total carbohydrates were calculated by difference. Energy was calculated according to the following equation: Energy $(\mathrm{kcal})=4 \times\left(\mathrm{g}_{\text {protein }}\right)+3.75 \times\left(\mathrm{g}_{\text {carbohydrate }}\right)+9 \times\left(\mathrm{g}_{\mathrm{fat}}\right)$

\subsubsection{Free sugars}

Free sugars were determined by high performance liquid chromatography coupled to a refraction index detector (HPLC-RI), using a previously described procedure (Heleno, Barros, Sousa, Martins, \& Ferreira, 2009). Data were analysed using Clarity 2.4 Software (DataApex). The compounds were identified by chromatographic comparisons with authentic standards. Quantification was performed using the internal standard (raffinose) method.

\subsubsection{Organic acids}

Organic acids were determined following a procedure previously optimized and described by the authors (Barros, Pereira, \& Ferreira, 2013). Detection was carried out in a PAD, using 215 and $245 \mathrm{~nm}$ as preferred wavelengths. The organic acids were quantified by comparison of the area of their peaks recorded at $215 \mathrm{~nm}$ with calibration curves obtained from commercial standards of each compound. Standards of organic acids (oxalic acid, quinic acid, citric acid and fumaric acid) were from Sigma Chemical Co. (St. Louis, MO, USA). The results were expressed in g per $100 \mathrm{~g}$ of dry weight (dw). 


\subsubsection{Phenolic compounds}

Phenolic compounds were determined in the UFLC system mentioned above, as previously described by the authors (Barros, Dueñas, Ferreira, Baptista, \& Santos-Buelga, 2009; Fernandes et al., 2013b). DAD detection was carried out using $280 \mathrm{~nm}$ and $370 \mathrm{~nm}$ as preferred wavelengths. The phenolic compounds were characterized according to their UV spectra and retention times, and comparison with authentic standards. For quantitative analysis, calibration curves were prepared from different standard compounds. Standards of phenolic compounds (protocatechuic, $p$-hydroxybenzoic and $p$-coumaric acids) and cinnamic acid were from Sigma Chemical Co. (St. Louis, MO, USA). The results were expressed in mg per $100 \mathrm{~g} \mathrm{dw}$.

\subsubsection{Fatty acids}

Fatty acids were determined by gas-liquid chromatography with flame ionization detection (GC-FID)/capillary column as described previously by the authors (Heleno, Barros, Sousa, Martins, \& Ferreira, 2009). Fatty acid identification was made by comparing the relative retention times of FAME peaks from samples with standards. The results were recorded and processed using the CSW 1.7 Software (DataApex 1.7, Prague, Czech Republic) and expressed in relative percentage of each fatty acid (obtained after Soxhlet extraction).

\subsubsection{Tocopherols}

Tocopherols were determined following a procedure previously optimized and described by the authors (Reis et al., 2012). The compounds were identified by chromatographic comparison with authentic standards. Quantification was based on the fluorescence signal response of each standard, using the IS (tocol) method and by using 
calibration curves obtained from commercial standards of each compound. The results were expressed in $\mu \mathrm{g}$ per $100 \mathrm{~g} \mathrm{dw}$.

\subsection{Antioxidant parameters}

\subsubsection{Extraction procedure}

The dried powder $(1 \mathrm{~g})$ was stirred with methanol $(30 \mathrm{~mL})$ at $25^{\circ} \mathrm{C}$ at $150 \mathrm{rpm}$ for $1 \mathrm{~h}$ and filtered through Whatman No. 4 paper. The residue was then extracted with an additional portion of methanol. The combined methanolic extracts were evaporated under reduced pressure (rotary evaporator Büchi R-210; Flawil, Switzerland), re-dissolved in methanol at $20 \mathrm{mg} / \mathrm{mL}$ (stock solution), and stored at $4{ }^{\circ} \mathrm{C}$ for further use. Successive dilutions were made from the stock solution and submitted to in vitro assays to evaluate the antioxidant activity of the samples (Fernandes et al., 2014)

\subsubsection{Antioxidant activity}

DPPH radical-scavenging activity was evaluated by using an ELX800 microplate reader (Bio-Tek Instruments, Inc; Winooski, VT, USA), and calculated as a percentage of DPPH discoloration using the formula: $\left[\left(A_{D P P H}-A_{S}\right) / A_{D P P H}\right] \times 100$, where $A_{S}$ is the absorbance of the solution containing the sample at $515 \mathrm{~nm}$, and $\mathrm{A}_{\mathrm{DPPH}}$ is the absorbance of the DPPH solution. Reducing power was evaluated by the capacity to convert $\mathrm{Fe}^{3+}$ into $\mathrm{Fe}^{2+}$, measuring the absorbance at $690 \mathrm{~nm}$ in the microplate reader mentioned above. Inhibition of $\beta$-carotene bleaching was evaluated though the $\beta$-carotene/linoleate assay; the neutralization of linoleate free radicals avoids $\beta$-carotene bleaching, which is measured by the formula: $\beta$-carotene absorbance after $2 \mathrm{~h}$ of assay/initial absorbance) $\times 100 \%$ (Fernandes et al. 2014). 


\subsection{Statistical analysis}

All the analyses (extractions) were performed in triplicate; each replicate was quantified also three times. Data were expressed as means \pm standard deviations. The fulfillment of the one-way ANOVA requirements, specifically the normal distribution of the residuals and the homogeneity of variance, was tested by means of the ShapiroWilk's, and the Levene's tests, respectively. For each parameter, significant differences among mean values were checked by Welch's statistics $(p<0.05$ means that the mean value of the evaluated parameter of at least one irradiation differs from the others). In the cases where statistical significance differences were identified, the dependent variables were compared using Tukey's honestly significant difference (HSD) or Tamhane's T2 multiple comparison tests, when homoscedasticity was verified or not, respectively.

Principal components analysis (PCA) was applied as pattern recognition unsupervised classification method. PCA transforms the original measured variables into new uncorrelated variables called principal components. The first principal component covers as much of the variation in the data as possible. The second principal component is orthogonal to the first and covers as much of the remaining variation as possible, and so on (Patras et al., 2011). The number of dimensions to keep for data analysis was evaluated by the respective eigenvalues (which should be greater than one), by the Cronbach's alpha parameter (that must be positive) and also by the total percentage of variance (that should be as higher as possible) explained by the number of components selected. The number of dimensions considered for PCA was chosen in order to allow meaningful interpretations, to ensure their reliability.

All statistical tests were performed at a 5\% significance level using the SPSS software, version 22.0 (IBM, Corp., Armonk, NY: USA). 


\section{Results and discussion}

\subsection{Effects on chemical parameters}

The nutritional parameters of $A$. caesarea and A. curtipes (Table 1) showed some relevant differences, despite belonging to the same genus, especially in what concerns water and fat contents. Nevertheless, water was the major component $(\approx 94 \%$ in $A$. caesarea; $\approx 84 \%$ in $A$. curtipes $)$ in fresh weight basis, while carbohydrates $(\approx 65 \%$ in $A$. caesarea; $\approx 62 \%$ in A. curtipes) were the major component per dry weight, followed by ash, proteins and fat contents. These nutritional profiles are very similar to those reported for A. caesarea and other species from this genus (Reis et al., 2011). In terms of the effects of EB irradiation, significant changes were detected in nearly all cases, except water content in A. caesarea. The highest doses, in particular, caused more significant effects, excluding the observed for energy (both mushrooms), water and fat contents (in $A$. curtipes). The highest differences among control and irradiated samples for proximate analysis were verified in protein content, which might be related to scission of the C-N bonds in the backbone of the polypeptide chain, or physical changes like unfolding (Molins, 2001), leading to a higher availability of nitrogen atoms with consequences in the Kjeldahl reaction, used for evaluating the nutritional value after the irradiation treatment.

Different free sugars profiles (Table 1) were detected for both Amanita. A. curtipes presented a higher content, with trehalose as the most abundant sugar followed by mannitol and fructose. In the extracts of $A$. caesarea, only mannitol and trehalose were detected, and in much lower quantities. The same sugars were previously detected in A. caesarea, but the amounts reported herein were lower (Reis et al., 2011), which might be explained by different maturity stages. Free sugars are among the most important parameters in quality assessment, since sugar content and composition can be lowered or modified by several conditions, like storage temperature, relative humidity, harvest time, oxygen level or 
packaging (Barreira, Pereira, Oliveira \& Ferreira, 2010). Samples submitted to EB irradiation presented significant changes in sugars contents, despite the produced effect had not been coherent in both species (sugars increase with irradiation in A. caesarea but not in A. curtipes). Irradiation is known for causing sugars degradation and, in this case, it might be hypothesized that some polysaccharide units have been hydrolysed releasing the corresponding free sugar units. Furthermore, the free sugar composition can also be influenced by different varieties, genotypes, ecological conditions, or technical practices (Barreira et al., 2010).

From Table 2, it is possible to conclude that the composition in organic acids is quite similar for both Amanita, with malic acid as the main organic acid, followed by fumaric acid and oxalic acid. The only detected phenolic acid was $p$-hydroxybenzoic acid, more than ten-fold higher in $A$. caesarea, which presented also cinnamic acid, contrarily to $A$. curtipes. Some slight differences, but statistically significant, were detected for the organic acids, despite lacking an identifiable tendency. Nevertheless, cinnamic acid suffered a strong decrease in irradiated samples.

Table 3 presents the individual fatty acids (FA) quantified above $0.2 \%$ in each mushroom species: C6:0, C8:0, C10:0, C12:0, C15:0, C17:0, C17:1, C18:3, C20:1, C20:2 (not detected in A. caesarea), C20:3+C21:0, C20:5, C22:0, C22:1 (not detected in $A$. curtipes), C23:0 and C24:1 (not detected in A. caesarea) were also quantified (and included further in the principal component analysis). The most abundant FA in these Amanita were palmitic acid (C16:0), oleic acid (C18:1) and linoleic acid (C18:2), as it is typical in this genus (Reis et al., 2011). Saturated fatty acids seemed to be more resistant to EB irradiation, while monounsaturated species tended to increase in percentage and polyunsaturated ones showed the opposite behavior, which is in agreement with its different radiosensitivity (Stewart, 2001). Even so, fatty acid percentages did not suffer any 
observable severe change, which might be explained by the fact that irradiation was performed in dried mushrooms. In fact, the general mechanism of lipids radiolysis (primary ionization, followed by migration of the positive charge either toward the carboxyl carbonyl group or double bonds) is more likely to occur in fresh mushrooms (Molins, 2001).

The tocopherols profiles (Table 4) revealed the presence of $\alpha$-, $\gamma$ - and $\delta$-tocopherol quantified in higher quantities in $A$. caesarea. $\gamma$-Tocopherol was the dominant form in both species, with $\alpha$-tocopherol as the minor isoform, in agreement with other reported results (Reis et al., 2011). In general, irradiated samples tended to present higher amounts, except for what was observed with $\gamma$-tocopherol in A. caesarea. This result should not, obviously, be interpreted as if irradiation causes an increase in tocopherol content but as a preservation effect of irradiation; on the other hand, the previously pointed out changes in the atmosphere of the samples containers might act by preventing the tocopherol degradation, due to a decrease in the molecular oxygen availability.

\subsection{Effects on antioxidant parameters}

The effects of using EB irradiation in the antioxidant activity of the Amanita extracts were evaluated by comparing the results obtained from different assays (Table 5). With no exception, irradiated samples (especially for the higher doses) showed to be more antioxidant, either as DPPH radicals scavengers, ferric reducers (conversion of a $\mathrm{Fe}^{3+} /$ ferricyanide complex to $\mathrm{Fe}^{2+}$ ) and $\beta$-carotene bleaching or TBARS formation inhibitors. The higher antioxidant activity was coherent with the levels of phenolic compounds, which also increased with extending irradiation doses. Except for TBARS formation inhibition, A. caesarea showed higher antioxidant activity than $A$. curtipes, and besides this action, the assayed mushroom proved to be particularly active as reducing 
agents. The increased TBARS formation inhibition might be related to the high amount of tocopherols (powerful lipophilic antioxidants) detected in the irradiated samples. The $\mathrm{EC}_{50}$ values are in general agreement with those reported in Amanita genus (Reis et al., 2011).

\subsection{Principal component analysis (PCA)}

In the former section, profiling changes resulting from EB irradiation were compared for each individual assayed parameter within each Amanita species. Despite the high number of detected statistically significant changes, it was not possible to present overall conclusions regarding the feasibility of this technology. Furthermore, it was intended to validate this technology independently of the mushroom species. Accordingly, in the present section the results were evaluated considering data for both species and considering all parameters simultaneously.

To conclude if EB irradiation allows maintaining the chemical and antioxidant profiles among non-irradiated and irradiated samples, principal components analysis (PCA) was applied to obtain output including the integrated effects on all parameters at once. Due to the variable magnitude between species for specific parameters (Tables 1-5), the values were normalized by subtracting the value corresponding to $0 \mathrm{kGy}$ to each of the values corresponding to 2, 6 and $10 \mathrm{kGy}$. The obtained differences were further divided by the value of the respective control. In this way, the classification procedure was applied to the differences caused by irradiation and not to the absolute values measured for each parameter. Due to practical reasons, only the parameters detected in both species were included in this analysis (fructose, C20:2, C22:1, C24:1 and cinnamic acid were excluded).

The plot of object scores (Figure 1) for EB irradiation dose showed that the first two dimensions (first: Cronbach's $\alpha, 0.964$; eigenvalue, 17.661; second: Cronbach's $\alpha, 0.946$; eigenvalue, 13.478) included most of the variance of all quantified variables $(37.6 \%$ and 
$28.7 \%$, respectively). The inclusion of a third dimension (Cronbach's $\alpha, 0.809$; eigenvalue, 4.802) would increase the percentage of explained variance with an additional $10.2 \%$, but the produced output would not allow such a meaningful interpretation as in the case of using two dimensions. Groups corresponding to each gamma irradiation dose ( $0 \mathrm{kGy}, 2$ kGy, $6 \mathrm{kGy}$ and $10 \mathrm{kGy}$ ) were not arranged individually, despite all the significant differences in Tables 1-5. This apparently random distribution seemed to be a direct consequence of the dissimilar effects of EB in each of the assayed species. As it can be depicted from Figure 1, only half of the object scores corresponding to $10 \mathrm{kGy}$ were grouped individually. These scores correspond with no doubt to A. caesarea, since they are characterized by high increase in ash, trehalose, mannitol and total sugars, and high decrease in carbohydrates, C16:1, C17:1, C18:3, reducing power and TBARS formation inhibition. However, the $10 \mathrm{kGy}$ dose had not the same effect on $A$. curtipes, leading to the grouping of its object scores together with those corresponding to samples irradiated with 2 and $6 \mathrm{kGy}$ (left side on the bottom). Accordingly, and despite the statistical significant changes identified for individual parameters, the effects of each EB dose do not seem to be distinguishable. All in all, when considering all nutritional, chemical and bioactive parameters at once, it does not seem to be relevant differences in using 2, 6 or $10 \mathrm{kGy}$.

\section{Conclusions}

The effects of different EB irradiation doses were assessed by measuring the changes produced on a wide variety of nutritional, chemical and antioxidant indicators from wild dried Amanita mushrooms. The evaluated profiles indicated differences among nonirradiated and irradiated samples, but the produced effect was often advantageous, as in the case of oleic acid or the improvement in the antioxidant activity. Furthermore, when considering all effects at once, it could be concluded that there are no relevant differences 
when using different irradiation doses. This was a very interesting finding, since it indicated the possibility of using the highest assayed dose (10 kGy), which has, off course, higher effectiveness from the decontamination and disinfestation point of view, without causing more pronounced effects than the lower doses. Accordingly, it would be advised to use the $10 \mathrm{kGy}$ dose, since the treated foods could also be available for persons with particular food safety concerns, and this dose did not cause stronger effects than the lower doses.

\section{Acknowledgements}

The authors are grateful to the Foundation for Science and Technology (FCT, Portugal) for financial support of research centres CIMO (PEst-OE/AGR/UI0690/2011) and REQUIMTE (PEst-C/EQB/LA0006/2011). Â. Fernandes and J.C.M. Barreira thank FCT, POPH-QREN and FSE for their grants (SFRH/BD/76019/2011 and SFRH/BPD/72802/2010, respectively). Prof. A. Chmielewski, General Director of the Institute of Nuclear Chemistry and Technology, Warsaw, Poland, for allowing e-beam irradiations.

\section{References}

AOAC. (1995). Official methods of analysis (16 ${ }^{\text {th }}$ Ed.). Arlington VA, USA: Association of Official Analytical Chemists.

Barreira, J.C.M., Pereira, J.A., Oliveira, M.B.P.P., \& Ferreira, I.C.F.R. (2010). Sugars profiles of different chestnut (Castanea sativa Mill.) and almond (Prunus dulcis) cultivars by HPLC-RI. Plant Foods for Human Nutrition, 65, 38-43. 
Barros, L., Dueñas, M., Ferreira, I.C.F.R., Baptista, P., \& Santos-Buelga, C. (2009). Phenolic acids determination by HPLC-DAD-ESI/MS in sixteen different Portuguese wild mushrooms species. Food and Chemical Toxicology, 47, 1076-1079

Barros, L., Pereira, C., \& Ferreira, I.C.F.R. (2013). Optimized analysis of organic acids in edible mushrooms from Portugal by ultra fast liquid chromatography and photodiode array detection. Food Analytical Methods, 6, 309-316.

Culleré, L., Ferreira, V., Venturini, M.E., Marco, P., \& Blanco, D. (2012). Evaluation of gamma and electron-beam irradiation on the aromatic profile of black truffle (Tuber melanosporum) and summer truffle (Tuber aestivum). Innovative Food Science and Emerging Technologies, 13, 151-157.

Ezekiel, C.N., Sulyok, M., Frisvad, J.C, Somorin, Y.M., Warth, B., Houbraken, J., Samson, R.A., Krska, R., \& Odebode, A.C. (2013). Fungal and mycotoxin assessment of dried edible mushroom in Nigeria. International Journal of Food Microbiology, 162, 231-236.

Fernandes, Â., Barreira, J.C.M., Antonio, A.L., Martins, A., Oliveira, M.B.P.P., \& Ferreira, I.C.F.R. (2013a). Combined effects of electron-beam irradiation and storage time on the chemical and antioxidant parameters of wild Macrolepiota procera dried samples. Food and Bioprocess Technology, 6, 2895-2903.

Fernandes, A., Barreira, J.C.M., Antonio, A.L., Santos, P.M.P., Martins, A., Oliveira, M.B.P.P., Ferreira, I.C.F.R. (2013b). Study of chemical changes and antioxidant activity variation induced by gamma-irradiation on wild mushrooms: comparative study through principal component analysis. Food Research International, 54, 18-25.

Fernandes, Â., Barreira, J.C.M., Antonio, A.L., Oliveira, M.B.P.P., Martins, A., \& Ferreira, I.C.F.R. (2014). Feasibility of electron-beam irradiation to preserve wild 
dried mushrooms: Effects on chemical composition and antioxidant activity. Innovative Food Science and Emerging Technologies, 22, 158-166.

Heleno, S. A., Barros, L., Sousa, M. J., Martins, A., \& Ferreira, I.C.F.R. (2009). Study and characterization of selected nutrients in wild mushrooms from Portugal by gas chromatography and high performance liquid chromatography. Microchemical Journal, 93, 195-199.

Heleno, S.A., Barros, L., Sousa, M.J., Martins, A., \& Ferreira, I.C.F.R. (2010). Tocopherols composition of Portuguese wild mushrooms with antioxidant capacity. Food Chemistry, 119, 1443-1450.

ICGFI - International Consultative Group on Food Irradiation (1999). In Facts about Food Irradiation. Buckinghamshire, United Kingdom.

ICMSF - International Commission on Microbiological Specifications for Foods (1985). Ecologia microbiana de los alimentos 2. Editorial Acribia, Zaragoza, Spain.

Lukasse, L.J.S., \& Polderdijk, J.J. (2003). Predictive modelling of post-harvest quality evolution in perishables, applied to mushrooms. Journal of Food Engineering, 59, 191-198.

Ma, L., Chen, H., Zhu, W., \& Wang, Z. (2013) Effect of different drying methods on physicochemical properties and antioxidant activities of polysaccharides extracted from mushroom Inonotus obliquus. Food Research International, 50, 633-640.

Patras, A., Brunton, N.P., Downey, G., Rawson, A., Warriner, K., \& Gernigon, G. (2011). Application of principal component and hierarchical cluster analysis to classify fruits and vegetables commonly consumed in Ireland based on in vitro antioxidant activity. Journal of Food Composition and Analysis, 24, 250-256.

Reis, F.S., Heleno, S.A., Barros, L., Sousa, M.J., Martins, A., Santos-Buelga, C., \& Ferreira, I.C.F.R. (2011). Toward the antioxidant and chemical characterization of 
mycorrhizal mushrooms from northeast Portugal. Journal of Food Science, 76, 824830.

Reis, F. S., Barros, L., Martins, A., \& Ferreira, I. C. F. R. (2012). Chemical composition and nutritional value of the most widely appreciated cultivated mushrooms: An interspecies comparative study. Food and Chemical Toxicology, 50, 191-197.

Rodríguez, J.A.S., Rodríguez, J.A., Prieto, O.G., Alfonso, A.T., Frade, B.L., Martín, E.A., \& Jarauta, T.P. (1991). El género Amanita Pers. Ex Hooker en la provincia de León. Acta Botánica Malacitana, 16, 123-132.

Şevik, S., Aktaş, M., Doğan, H., \& Koçak, S. (2013). Mushroom drying with solar assisted heat pump system. Energy Conversion and Management, 72, 171-178.

Shephard, G.S. (2008). Impact of mycotoxins on human health in developing countries. Food Additives and Contaminants: Part A, 25, 146-151.

Stewart, E.M. (2001). Food Irradiation Chemistry. Food irradiation: Principles and applications. R. A. Molins. New York, USA, John Wiley \& Sons, 37-76.

Supriya, P., Sridhar, K.R, Nareshkumar, S., \& Ganesh, S. (2012). Impact of electron beam irradiation on fatty acid profile of Canavalia Seeds. Food and Bioprocess Technology, 5, 1049-1060.

\section{Figure Legends}

Figure 1. Biplot of object (electron-beam irradiation doses) scores and component loadings (evaluated parameters). 